\title{
Selective media for isolation of Burkholderia (Pseudomonas) cepacia from the respiratory secretions of patients with cystic fibrosis
}

\author{
N Cimolai, C Trombley, A G F Davidson, L T K Wong
}

\begin{abstract}
One hundred and six specimens from 90 patients with cystic fibrosis were evaluated for the presence of Burkholderia cepacia using a current routine diagnostic protocol as well as a research protocol involving polymyxin B-MacConkey agar without crystal violet, $P C$ agar, OFPVL agar, and a selective brain-heart infusion broth. Ten specimens from eight patients $(8.9 \%)$ were positive by any method. The selective enrichment broth was the only medium that yielded $B$ cepacia from all 10 positive samples, although the routine protocol was successful for eight of these. Transient carriage was identified in one patient. Epidemiological studies may be better served by the use of selective enrichment rather than selective solid media alone. Carrier status for $B$ cepacia requires more strict definition if positive carrier status is to be accepted as having medical importance. (F Clin Pathol 1995;48:488-490)
\end{abstract}

Keywords: Burkholderia cepacia, cystic fibrosis.

Burkholderia (Pseudomonas) cepacia colonisation and pulmonary infection in patients with cystic fibrosis are often markers of chronicity. ${ }^{1}$ Nevertheless, the association, proposed by some, between the presence of this bacterium and accentuated deterioration of pulmonary function has led clinicians to believe that definitive knowledge of the organism's presence is essential. ${ }^{2}$ Presence of this bacterium is also associated with the failure of lung transplantation in these patients ${ }^{3}$ and there is growing concern over the potential for person to person transmission of $B$ cepacia; the latter has resulted in the proposal that knowledge of carrier status is essential in maintaining appropriate infection control practices.

Given the complex microbiology of cystic fibrosis sputa, it has been suggested that the use of selective media will be important for maximising the determination of $B$ cepacia carrier status. Several previous studies have examined the development and clinical use of selective and differential media. ${ }^{5-8}$ There is some evidence that laboratories may under- estimate the frequency of $B$ cepacia by using otherwise standard microbiological media and techniques. ${ }^{9}$ In this study, we determined whether the use of selective and enrichment media could enhance the recovery of $B$ cepacia from specimens which were acquired from a population previously designated to be of low prevalence for carriage.

\section{Methods}

One hundred and six respiratory specimens were acquired from 90 patients who attended the cystic fibrosis clinic at the British Columbia's Children's Hospital, Vancouver. Repeat specimens were acquired from some patients who attended the clinic a second time during the study period, but these were obtained no sooner than two months after the initial sampling. The specimens included 35 throat swabs (33\% of the total) and 71 sputa $(67 \cdot 0 \%)$. Throat swabs were collected when the collection of sputa was impracticable (for example, because the child was too young). The frequencies of the patients according to age group were: 0 to 5 years, $30 \%$; 6 to 10 years, $22 \cdot 2 \%$; 11 to 15 years, $28.9 \%$; and 16 to 18 years, $18.9 \%$.

The respiratory specimens were evaluated by both the routine diagnostic laboratory and a research technologist. In the diagnostic laboratory, equal portions of sputa were inoculated by standard techniques onto media including sheep blood agar, chocolate, mannitol salt, and MacConkey agars. These solid media were incubated for a minimum of 24 hours before bacterial growth was assessed. Morphologically variant colonies were closely examined and subcultured for another 18 hours onto sheep blood agar. In order to maximise the finding of $B$ cepacia and variable strains of $P$ s aeruginosa or other pseudomonads, considerable attention was paid to the colonial morphology. A preliminary classification of phenotype was made according to the following descriptions: classic, mucoid, gelatinous, enterobacteria-like, dwarf, and rough. ${ }^{10}$ The research technologist assessed the culture yield on other media while being blinded to the results from the routine laboratory. Selective and enrichment media included: MacConkey (oxoid) without crystal violet but 
containing $30 \mathrm{mg} / \mathrm{l}$ of polymyxin $\mathrm{B}$, OFPVL agar $^{5}$ (original formulation but containing $15 \mathrm{mg} / \mathrm{l}$ of vancomycin rather than bacitracin), PC agar $^{6}$ (commercially acquired; Prepared Media Laboratories), and brain-heart infusion broth with polymyxin B $(30 \mathrm{mg} / \mathrm{l})$, vancomycin $(15 \mathrm{mg} / \mathrm{l})$, and amphotericin B $(1 \mathrm{mg} / \mathrm{l})$. Equivalent portions of sputa were inoculated onto the experimental media. Throat swabs were immersed into $0.5 \mathrm{ml}$ of phosphate buffered serum ( $\mathrm{pH} \mathrm{7 \cdot 2)}$ and equal portions of the diluent were then inoculated onto the solid media or into the broth. All media were incubated for up to 72 hours, although generally inspected at 24 hour intervals. The selective enrichment broth was subcultured onto a sheep blood agar and an OFPVL plate at 24 hours. Standard biochemical criteria using replicator agar plates or commercial biochemical strips (API NFT, Bio Merieux) or both were used to define $B$ cepacia.

\section{Results}

Serial dilutions of $B$ cepacia laboratory suspensions (five different strains), followed by quantitative studies, indicated that 1-5 colony forming units could be detected by the enrichment process.

Ten $(9 \cdot 4 \%)$ respiratory specimens yielded $B$ cepacia and the 10 positive specimens were obtained from eight patients $(8 / 90 ; 8 \cdot 9 \%)$. Two patients were found to carry $B$ cepacia on two different occasions. One patient yielded $B \mathrm{ce}$ pacia on the first sampling but not on follow up. At most, two specimens were collected from any one patient.

All 10 specimens which were positive for $B$ cepacia were identified by the use of the brain-heart infusion enrichment process. The table illustrates the frequency of isolation with each medium. The one patient who was negative for $B$ cepacia on routine culture but positive on all four selective media was known to have been positive by the routine diagnostic procedure on a previous occasion before this study. The mean age for patients with $B$ cepacia was $15 \cdot 5$ years (range $10-18$ years), while the mean age for patients who did not carry this bacterium was 9.6 years (range 4 months-18 years). Both patients who were found to be culture positive by the non-routine selective media were 17 years of age. The majority of culture methods yielded moderate to heavy growths of $B$ cepacia, with some exceptions. The single specimen which was negative by polymyxin B-MacConkey, OFPVL, and PC agars, yielded only a light growth with the routine examination and a heavy growth after enrichment; this specimen was a throat swab.

Frequency of $B$ cepacia isolation from varied selective and enrichment processes

\begin{tabular}{llllll}
\hline $\begin{array}{l}\text { No. of } \\
\text { specimens }\end{array}$ & $\begin{array}{l}\text { Diagnostic } \\
\text { bench }^{\text {a }}\end{array}$ & Mac & PC & OFPVL & BHI $^{\mathrm{b}}$ \\
\hline 7 & + & + & + & + & + \\
1 & + & + & + & + & + \\
1 & - & + & + & + & + \\
1 & - & + & + & + \\
\hline
\end{tabular}

Nomenclature and composition of media are detailed in Methods.

${ }^{b}$ Brain-heart enrichment broth.
PC agar appeared to be more inhibitory to $B$ cepacia than polymyxin B-MacConkey or OFPVL; four out of nine specimens positive on PC agar yielded light growths while the other selective agars contained moderate to heavy growths. Suppression of flora other than $B$ cepacia occurred the least on polymyxin BMacConkey. Fungi were among the more common contaminants of all media. The antifungal component of the enrichment was helpful although not entirely suppressive for all specimens, and thus the addition of a greater concentration of this component might be warranted. The unique colonial phenotype of $B$ cepacia on OFPVL was found to facilitate the identification process.

\section{Discussion}

The frequency of $B$ cepacia from our cystic fibrosis population is lower than has been reported from Toronto, Canada, and is approximately the same as the average rate of colonisation previously reported in other North American centres. ${ }^{5}$ Our lower frequency may, however, be somewhat biased by the lack of any adult cystic fibrosis patients in this study population.

The use of selective media did not dramatically increase the perceived carriage rate. Eight of 10 carriers were positive by the routine method and one patient was intermittently identified before by the current routine method, although not on this occasion. Thus the real increment in the identification of carriage might only have been $10 \%$. In the context of several pseudomonad colony phenotypes on primary culture (that is, possibly $B$ cepacia, varied $P S$ aeruginosa strains, and other non-fermentative bacteria), it is not surprising that $B$ cepacia might not be found, especially when the organism exists in lesser numbers than the other competing pseudomonads. We believe that our critical examination for phenotypic colonial variants may maximise the detection of $\mathrm{B} \mathrm{ce}$ pacia, in contrast to a more cursory routine examination. In a context where selective media are not used, our present approach is important given that the MacConkey medium with crystal violet is recognised as being relatively inhibitory to $B$ cepacia (our experience; also ${ }^{689}$ ).

PC agar did not confer any advantage over the other selective media and was relatively inhibitory, as assessed by semi-quantitative growth of $B$ cepacia. Other studies do not support these findings, ${ }^{7-9}$ but do agree that PC agar is effective in suppressing other bacterial species. Selective MacConkey without crystal violet did not appear to inhibit $B$ cepacia, although it does when used with crystal violet, but there did not appear to be any major advantages of the former. The implementation of any selective or enrichment medium should in part depend upon the degree to which isolation is enhanced. It is conceivable that the use of selective or enrichment media might be restricted to the patient population which is at greater risk (that is, older patients). In our population, all $B$ cepacia carriers were older than 9 years. 
Previous studies have focused predominantly on selection alone rather than on selection with enrichment. As for other bacterial searches, it would be expected that some form of selective enrichment should perform the best. The stringency of search for $B$ cepacia is, of course, the driving force for the need for selective or enrichment techniques. For the purposes of antimicrobial chemotherapy, the finding of lesser quantities of $B$ cepacia compared to other pseudomonads may not alter a treatment regimen. In contrast, the belief that person to person transmission does occur with reasonable frequency has caused some to believe that absolute knowledge of carrier status is essential.

How should the carrier be defined and will all such carriers confer equal risk for transmission? If the simple identification of $B$ cepacia at any site, regardless of quantity, defines the carrier, how many samples (and from which sites) should be acquired? If a carrier is identified, what criteria should be present before the patient ceases to be classified as a carrier? Our experience with the finding of transient oropharyngeal carriage despite the use of selective enrichment in one patient is a good example of a circumstance where the above dilemmas need to be resolved. Transient carriage of $B$ cepacia after acquisition from summer camp exposure is known to occur. Although it is sensible to observe good personal and interpersonal hygiene so that respiratory pathogens in general, let alone $B$ cepacia, are not transmitted, the strict definition of a carrier and the social segregation that can arise, may not always be in the best interests of the patient. Studies which examine these issues may be better served by the use of selective enrichment media.

1 Tablan OC, Martone WJ, Doershuk CF, et al. Colonization of the respiratory tract with Pseudomonas cepacia in cystic f i the

2 Isles A, Maclusky M, Corey R, et al. Pseudomonas cepacia infections in cystic fibrosis; an emerging problem. $\mathcal{F}$ Pediatr infections in cystic

3 Snell GI, DeHoyos A, Krajden M, Winter T, Maurer JR Pseudomonas cepacia in lung transplant recipients with cystic fibrosis. Chest 1993;103:466-71.

4 Govan JRW, Brown PH, Maddison J, et al. Evidence for transmission of Pseudomonas cepacia by social contact in cystic fibrosis. Lancet 1993;342:15-19.

5 Welch DF, Muszynski MJ, Pai CH, et al. Selective and differential medium for recovery of Pseudomonas cepacia from the respiratory tracts of patients with cystic fibrosis. 7 Clin Microbiol 1987;25:1730-4.

6 Gilligan PH, Gage PA, Bradshaw LM, Schidlow DV, DeCicco BT. Isolation medium for the recovery of Pseudomonas cepacia from mistory monas cepacia from respiratory secretions of patients with

Black-Payne C, Lierl MB, Bocchini JA, Hilman BC, Rambin $\mathrm{ED}$, Silberman R. Comparison of two selective media developed to isolate Pseudomonas cepacia from patients developed to isolate Pseudomonas cepacia from patients with cys

8 Carson LA, Tablan OC, Cusick LB, Jarvis WR, Favero MS, Bland LA. Comparative evaluation of selective media for isolation of Pseudomonas cepacia from cystic fibrosi patients and environmental sources. F Clin Microbiol 1988; 26:2096-100.

9 Tablan OC, Carson LA, Cusick LB, Bland LA, Martone WJ, Jarvis WR. Laboratory proficiency test results on use of selective media for isolating Pseudomonas cepacia from isolating Pseudomonas cepacia from simulated sputum specimens of patients with cystic fib-

10 Wahba AH, Darrell JH. The identification of atypical strain of Pseudomonas aeruginosa. $\mathcal{F}$ Gen Microbiol 1965;38:32942 .
Hepatitis and

Retrovirus Laboratory,

PHLS Virus Reference

Division,

Central Public

Health Laboratory,

61 Colindale Avenue,

London NW9 5HT

L McAlpine

J V Parry

P P Mortimer

Department of

Medical Microbiology,

Charing Cross and

Westminster Medical

School,

369 Fulham Road,

London

SW10 9NH

D Shanson

Correspondence to:

Dr P P Mortimer.

Accepted for publication

26 September 1994

L McAlpine, J V Parry, D Shanson, P P Mortimer

Abstract

The sensitivity of 12 commonly used antiHIV-1/HIV-2 enzyme immunoassays was evaluated. The assays, each of which utilises at least one synthetic HIV antigen, were tested against a panel of 1092 specimens previously designated anti-HIV positive. In a total of 13104 tests there were eight false negative results attributable to assay insensitivity: three were on two serum samples collected close to seroconversion and five on another serum specimen. These eight false negative results arose in seven different assays. Five other false results were attributable to technical error. This false negativity rate indicates that all of the assays performed adequately and leads to an estimate of one false negative result in a thousand tests in routine diagnostic practice. Because of the antigenic heterogeneity of HIV strains, similar evaluations would be required in several regions before this satisfactory level of sensitivity in anti-HIV assays incorporating synthetic antigens could be said to be universal.

(f Clin Pathol 1995;48:490-493)

Keywords: HIV screening, synthetic HIV antigens, false negative results. 